\title{
Improved joint preventive maintenance and hedging point policy
}

\section{F. Berthaut ${ }^{\text {a }}$, A. Gharbi ${ }^{\text {a, }}$, J.-P. Kenné ${ }^{b}$ and J.-F. Boulet ${ }^{c}$}

\footnotetext{
a Automated Production Engineering Department, École de technologie supérieure, Production System Design and Control Laboratory, University of Québec, 1100 Notre-Dame Street West, Montreal, Que., Canada H3C 1K3.

${ }^{\mathrm{b}}$ Mechanical Engineering Department, École de technologie supérieure, University of Québec, 1100 Notre-Dame Street West, Montreal, ,Que., Canada H3C 1K3.

c TRELLISYS TECHNOLOGIES, INC. 755, boul. Saint-Jean, bureau 400 Pointe-Claire, Que., Canada H9R 5M9.
}

\section{Abstract:}

We investigate the preventive maintenance and inventory control problem of a one-machine, oneproduct manufacturing system subject to random breakdowns. Both preventive and corrective interventions have random and non-negligible durations during which an excess of final products inventory is necessary to fulfill demand. The objective of this study is to find the production rate and the preventive maintenance schedule that minimize the total cost of maintenance and inventory/backlog in the case of periodic preventive maintenance. A near-optimal policy characterization with a simple structure is carried out using a numerical approach. Such a policy is a combination of a hedging point policy and a modified periodic preventive maintenance strategy, under which preventive maintenance actions are performed only if the inventory level exceeds a sufficient level. A simulation-based experimental approach is adopted to achieve a close approximation of the optimal control parameters. It is concluded from a sensitivity analysis and a comparative analysis that the near-optimal control policy leads to a significant cost reduction as compared to the combination of a hedging point policy and a classical periodic preventive maintenance policy.

Keywords: Preventive maintenance; inventory control; stochastic dynamic programming; simulation; response surface methodology. 


\section{Introduction}

Operations planning is critical for the proper functioning of a firm (Karen et al., 2003). Planning requires making decisions based on a large amount of information concerning customer demand, the production process, raw material supply, failures and preventive maintenance, which may be sources of uncertainties that the system must deal with in order to enhance profitability. This paper aims to present a better insight of the interaction between failures and preventive maintenance with inventory in a manufacturing system in which repair and maintenance durations are random and non-negligible.

Barlow and Hunter (1960) and Barlow and Proschan (1965) introduced the basis of the two main preventive maintenance (PM) strategies: the age replacement policy (ARP), which consists in performing PM whenever the age of the unit reaches a predefined age threshold, and the block replacement policy (BRP), which calls for PM to be performed at regular intervals. Under both the ARP and the BRP, units are replaced as failures occur (corrective maintenance, CM). The above authors showed that the ARP is better than the BRP from an economic point of view, in the sense that the BRP can result in the replacement of relatively new units. However, the ARP is more difficult to implement and manage than the BRP because the ARP requires tracking the ages of units and involves modifying PM planning after each maintenance action. An interesting improvement of these policies proposed by Berg and Epstein (1976) and Archibald et al. (1996) is based on the BRP, but involves the skipping PM of components whose age falls below a certain threshold. This policy retains the advantages of both the BRP and the ARP and results in maintenance costs that are slightly higher than with the ARP. These maintenance models assume that $\mathrm{CM}$ and PM are performed instantaneously. In most cases, maintenance durations are nonnegligible and production interruptions due to maintenance require times to repair that may lead to inventory shortages and penalty costs. With respect to these close interactions, it would be interesting to address such control problems through combined preventive maintenance and inventory policies.

Several authors have broached the problem of preventive maintenance and buffer inventory control. The evolution of the inventory level during a maintenance cycle with non-zero maintenance times has been analysed in order to determine and minimize the exact overall cost. The mathematical models used in the literature to control maintenance activities are based either on a BRP (Ki Ling et al. 1997; Chelbi and Ait-Kadi, 2004) or on an ARP (Gharbi et al. 2007; Rezg et al. 2008). These preventive maintenance strategies are combined with an inventory control policy that consists in building up and maintaining a buffer stock to respond to demand during maintenance operations. However, the optimality of the structure of such joint control policies has not been established, and the authors' works are limited by restrictive assumptions, such as the absence of breakdowns during stock build-up periods, the loss of unmet demands during repair periods, or inventory levels being set to zero after maintenance activities, as mentioned in Rezg et al. (2008) and stipulated in references herein. As well, this approach is limited by the calculation of convolution products representing the sum of random variables (i.e., time between failures plus CM duration), which are hard to express for most probability distributions (Chelbi and Ait-Kadi, 2000).

Markov decision models have also proved successful in solving the maintenance control problem of a deteriorating machine, inspected at discrete time epochs, that provides a downstream buffer. For a predefined inventory policy, similar to that previously mentioned, Van der Duyn Schouten and Vanneste (1995), Kyriakidis and Dimitrakos (2006) and Dimitrakos and 
Kyriakidis (2008) showed the optimality of control-limit type policy, such that PM is performed if the degree of deterioration exceeds a critical level that depends on the buffer content.

Joint consideration of production planning and corrective maintenance problems in flexible manufacturing systems has been tackled using the optimal control theory (Rishel, 1975; Kimemia and Gerschwin, 1983), which has culminated in the hedging point policy (HPP) concept (Akella and Kumar, 1986). Within such a policy, a finished goods inventory surplus is maintained during times of excess capacity in order to deal with future interruptions and possible shortages due to machine breakdowns. The optimality of the HPP has been demonstrated for failure and repair times described by homogeneous Markov processes (i.e., constant transition rates), and therefore, for a failure replacement maintenance strategy. For general machine up and down times, several extensions have been proposed in order to increase the system capacity with either CM or PM or both (Kenné and Nkeungoue, 2008). On the one hand, CM has been considered by controlling the repair rate, with failure models which are not age dependent (Kenné et al. 2003; Pellerin et al. 2007). On the other, PM has been introduced, in the case of an increasing failure rate, with agedependent control policies derived from the HPP and the ARP (Boukas and Haurie, 1990; Kenné and Gharbi, 1999; Gharbi and Kenné, 2000). More specifically, PM is skipped if the inventory level is below the hedging point in Kenné and Gharbi (1999) and in Gharbi and Kenné (2000). An interesting way to proceed would be to control PM interventions with BRP instead of ARP, since BRP does not require a tracking of the deterioration of the machine, and is easier to control.

Primarily, this paper addresses joint maintenance and inventory control problems in the case of periodically scheduled PM (BRP) during which maintenance can be skipped. This is motivated by the ease with which a BRP is planned and managed. Furthermore, the idea of skipping PM is based on the modified BRP proposed by Berg and Epstein (1976) and Archibald and Dekker (1996) in order to avoid consecutive failures and PM, which lead to a waste of components and a risk of shortage. We also relax the restrictive assumptions commonly used in preventive maintenance and buffer inventory control with regards to the occurrence of breakdowns during stock build-up periods, the loss of unmet demand, and the periodicity of the inventory trajectory. The problem is addressed as an optimal control problem, and our main contribution lies in providing a near-optimal joint control policy. First, the characterization of the near-optimal joint control policy is obtained through a numerical approach. A close approximation of the associated control parameters is then achieved using a flexible simulation-based experimental approach. A near-optimal joint control policy is thus completely determined, and a comparison is made with the combination of the HPP and classical BRP.

The paper is organized as follows: Section 2 states the preventive maintenance and inventory control problem. Numerical methods are then used in Section 3 to carry out a characterization of the near-optimal policy with a simple structure described by several control parameters. The optimal control parameters and the incurred cost are obtained through a simulation-based experimental approach described in Section 4 and illustrated in Section 5 using an example. Subsequently, a sensitivity analysis of the control policies obtained with regards to the cost parameters is presented in Section 6. The control policies are compared in Section 7 for a wide range of time and cost configurations, and finally, concluding remarks are given in Section 8 .

\section{Control problem statement}

We consider the simultaneous control of the production and maintenance activities of a failureprone facility producing one part type. There is a transition from operational mode (OP) to PM 
each $T$ units of time, while the transition from OP to a failure mode (CM) randomly depends on the increasing failure rate distribution of the considered production system. In the corresponding transition diagram, a PM can be performed right after a CM, given that PM activities are scheduled at fixed time periods (i.e., at each $k T, k=1,2,3 \ldots$ ). From a practical point of view, a machine may have multiple operational states, as illustrated in Figure 1, where the operational mode, $\mathrm{OP}$, is considered as a set of $N$ sub-states (i.e., $O P=\left\{O P_{i}, i=1,2, \ldots, N-1, N\right\}$ ). The dynamic of the machine is characterized by transitions from mode $\mathrm{OP}_{\mathrm{i}}$ to mode $\mathrm{OP}_{\mathrm{i}+1}$ for various stages of degradation, and from $\mathrm{OP}_{\mathrm{i}}$ to $\mathrm{CM}$ or to $\mathrm{PM}$.

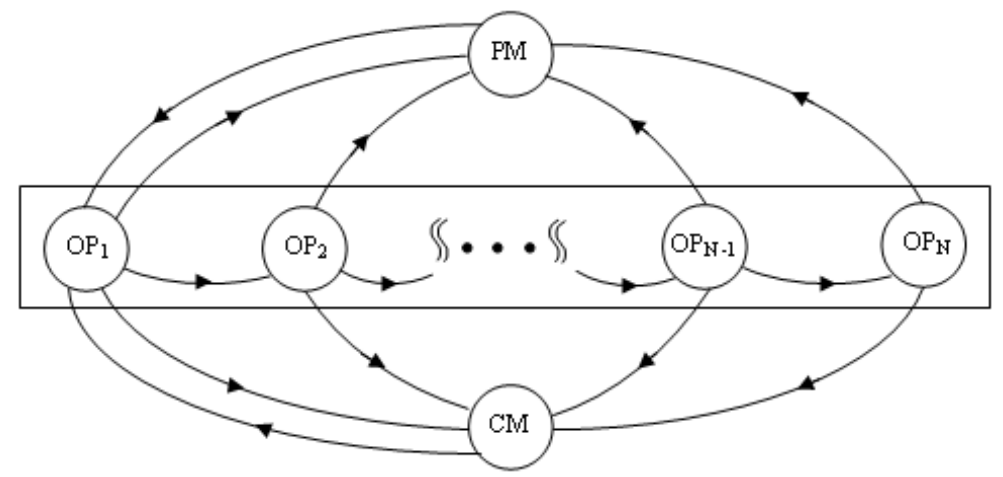

Figure 1 States transition diagram with multiple operational sub-states

In such a model, the transition rates from $\mathrm{OP}_{\mathrm{i}}$ to $\mathrm{PM}$ were considered to be the control variables, $\omega_{i}, i=1,2, \ldots, N$, which allow for the possibility of operation-dependent or timedependent PM actions. Hence, the PM frequency increases in time when going from operational modes $\mathrm{OP}_{\mathrm{i}}$ to $\mathrm{OP}_{\mathrm{i}+1}$. The discrete dynamics of the system (machine states) is described by a stochastic process, $\{\alpha(t), t \geq 0\}$, with values in $B=\{1,2, \ldots, N-1, N, N+1, N+2\}$ such that:

$$
\alpha(t)= \begin{cases}1 & \text { if the machine is in operational mode } \mathrm{OP}_{1} \\ 2 & \text { if the machine is in operational mode } \mathrm{OP}_{2} \\ \vdots & \text { if the machine is in operational mode } \mathrm{OP}_{\mathrm{N}-1} \\ N & \text { if the machine is in operational mode } \mathrm{OP}_{\mathrm{N}} \\ N+1 & \text { if the machine is under corrective maintenance } \\ N+2 & \text { if the machine is under preventive maintenance }\end{cases}
$$

The continuous dynamics of the inventory/backlog level, $x(t)$, is given by:

$$
\frac{d}{d t}(x(t))=u(t)-d, x(0)=x_{0}
$$

where $u(t)$ is the production rate at time $t, d$ is the demand rate and $x_{0}$ is the initial stock level.

The set of admissible decisions $(u, \omega)$ is given by: 


$$
\Gamma(\alpha)=\left\{(u, \omega) \mid 0 \leq u \leq u_{\max }, 0 \leq \omega_{i} \leq \omega_{\max }\right\}
$$

where $u_{\max }$ and $\omega_{\max }$ denote the maximum production rate and the PM rate, respectively.

The instantaneous maintenance and inventory total cost, $g($.$) , is given by the following$ equation:

$$
g(x, \alpha)=c^{+} \cdot x^{+}(t)+c^{-} \cdot x^{-}(t)+c_{1} \cdot I_{C M}^{+}(t)+c_{2} \cdot I_{P M}^{+}(t),
$$

where $c^{+}$is the inventory holding cost per time unit per item, $c^{-}$is the backlog cost per time unit per item, $c_{1}$ is the CM cost, $c_{2}$ is the PM cost, $x^{+}(t)=\max (0, x(t)), x^{-}(t)=\max (-x(t), 0)$ and

$$
I_{C M}^{+}(t)=\left\{\begin{array}{ll}
1 & \text { if } \alpha(t)=N+1 \\
0 & \text { otherwise }
\end{array}, I_{P M}^{+}(t)=\left\{\begin{array}{ll}
1 & \text { if } \alpha(t)=N+2 \\
0 & \text { otherwise }
\end{array} .\right.\right.
$$

The overall infinite horizon discount cost, $J($.$) , is given as:$

$$
J(x, \alpha, u, \omega)=E\left[\int_{0}^{\infty} e^{-\rho \cdot t} \cdot g(t) \mid x(0)=x, \alpha(0)=\alpha\right]
$$

where $\rho$ is the discount rate and $(x, \alpha)$ are the initial conditions on the state variables.

The value function $v(x, \cdot)$, given the initial state of the system $(x, \alpha)$, is:

$$
v(x, \alpha)=\inf _{(u, \omega) \in \Gamma(\alpha)} J(x, \alpha, u, \omega)
$$

Based on the state transition diagram in Figure 1, it can readily be shown, under appropriate regularity conditions imposed on the control, similar to those in Akella and Kumar (1986), that $v(x, \alpha)$ is the solution of the following HJB equations:

$$
\rho \cdot v(x, \alpha)=\min _{(u, \omega) \in \Gamma(\alpha)}\left\{(u-d) \cdot v_{x}(x, \alpha)+Q \cdot v(x, \cdot)(\alpha)+g(x, \alpha)\right\}, \forall x \in R, \forall \alpha \in B
$$

where $v_{x}(x, \alpha)$ is the partial derivative of the value function and $Q$ is the infinitesimal generator (or matrix of transition rates) of the stochastic process $\alpha(t)$.

Note that for a large number of modes (i.e., $N \rightarrow \infty$ ), it is impossible to solve these equations either analytically or numerically in order to obtain the value function and the associated control policy.

\section{Approximated structure of the optimal control policy}

For a finite number of modes, the numerical methods based on Kushner's approach are used to solve HJB equations (Kushner and Dupuis, 1992). The solution of the numerical approximation of the function $v(x, \alpha)$ may be obtained either by successive approximation or using policy improvement techniques (Boukas and Haurie, 1990; Kushner and Dupuis, 1992). The approximation consists in bounding the state variables space with a large domain and meshing this space with a finite grid of discrete values of the state variables. If $v_{h}(x, \alpha)$ is an approximation of $v(x, \alpha)$, its partial derivative and equations (5) can then be expressed as a function of $v_{h}(x, \alpha)$ and 
of the step of the grid $h$. Subsequently, $v_{h}(x, \alpha)$ is obtained by solving the modified HJB equations with the appropriate boundary conditions (Kenné et al., 2003).

From the structure of the HJB equations and their corresponding solutions for $N=10$, we obtain the control policy illustrated in Figure 2. To ensure a clear characterization of the control policy, the production and maintenance policies are each observed separately. The production rate is illustrated in Figure 2(a) and 2(b), and the PM rate in Figure 2(c) and 2(d), for two different values of the backlog cost. Specifically, Figure 3 shows the corresponding production and $\mathrm{PM}$ rates at operational modes $\mathrm{OP}_{3}$ and $\mathrm{OP}_{7}$ with $u_{\max }=1, d=0.9$ and $\omega_{\max }=0.75$, $M T T C R=10$ (mean time to perform $\mathrm{CM}$ ), MTTPM $=1$ (mean time to perform PM), $c^{+}=1$, $c_{1}=20, c_{2}=10$ and $c^{-}=10$ (for Figures 2(a), 2(c), 3(a) and 3(c)) and $c^{-}=200$ (for Figures 2(b), 2(d), 3(b) and 3(d)).

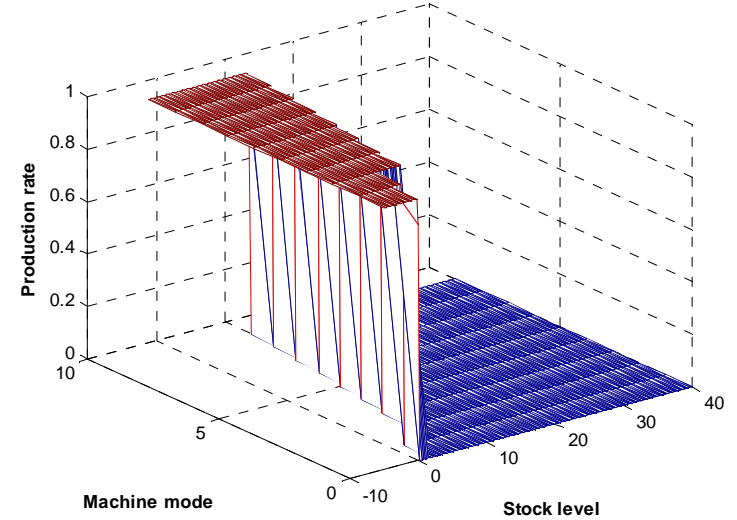

(a)

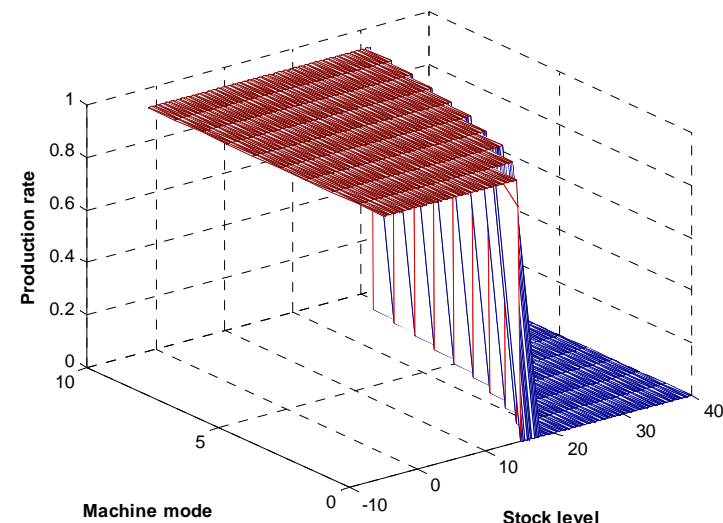

(b)

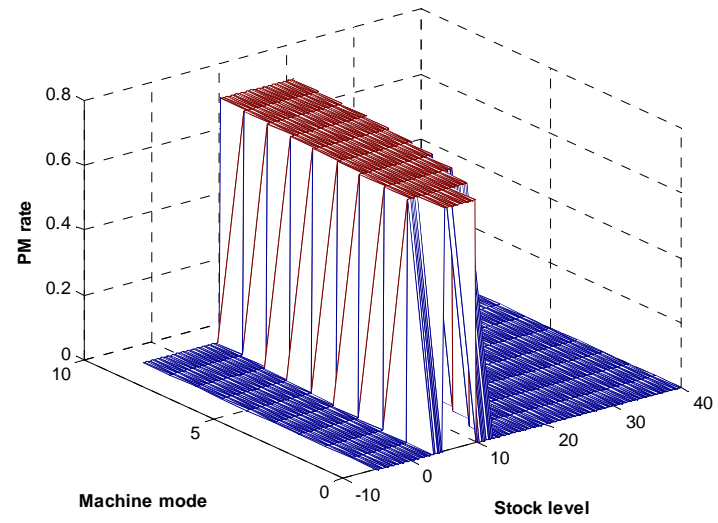

(c)

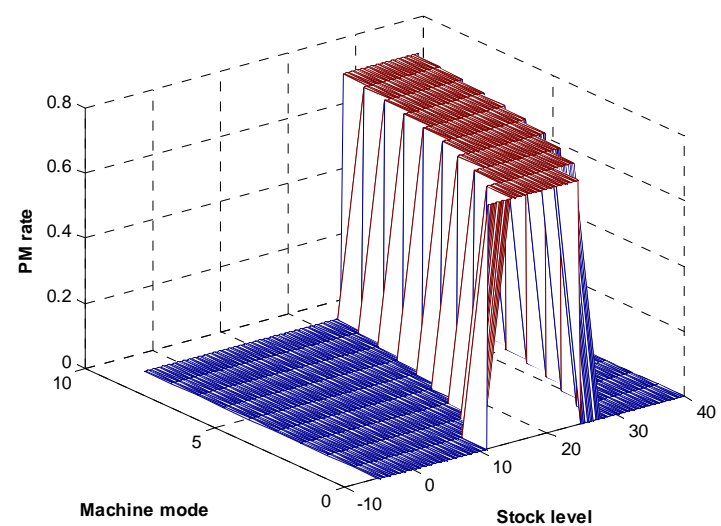

(d)

Figure 2 Production and PM rates 


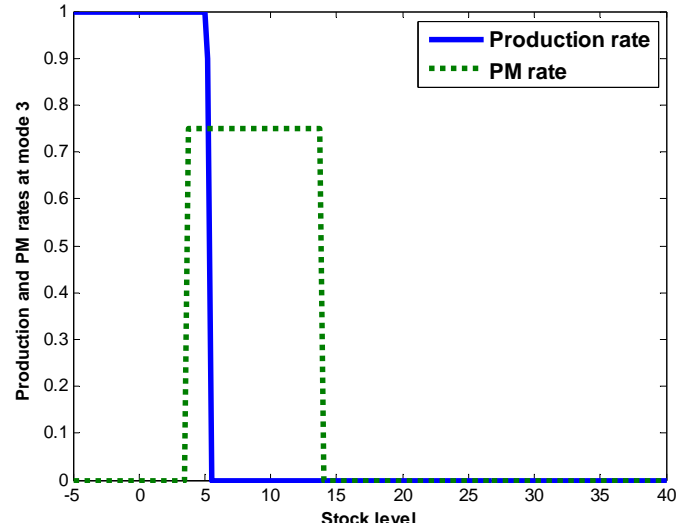

(a)

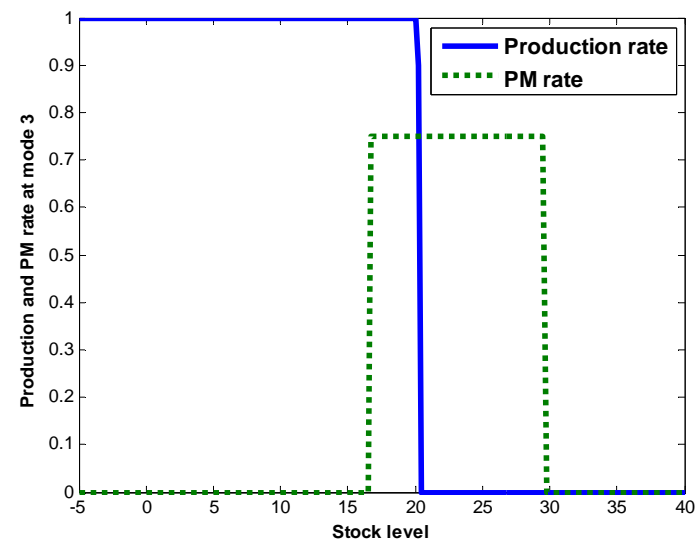

(b)

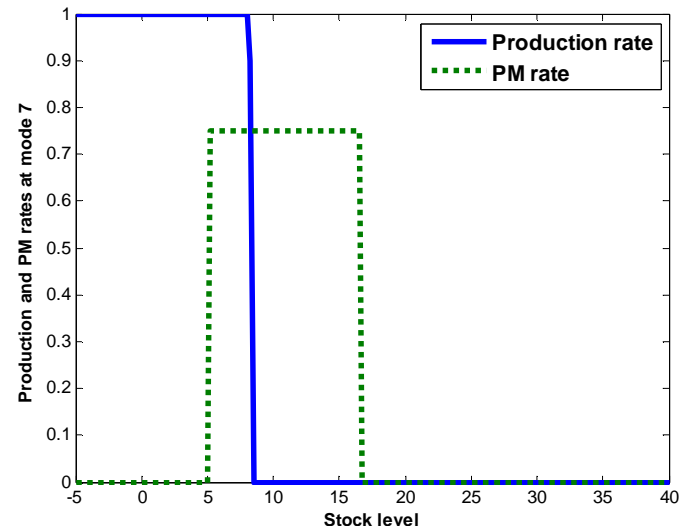

(c)

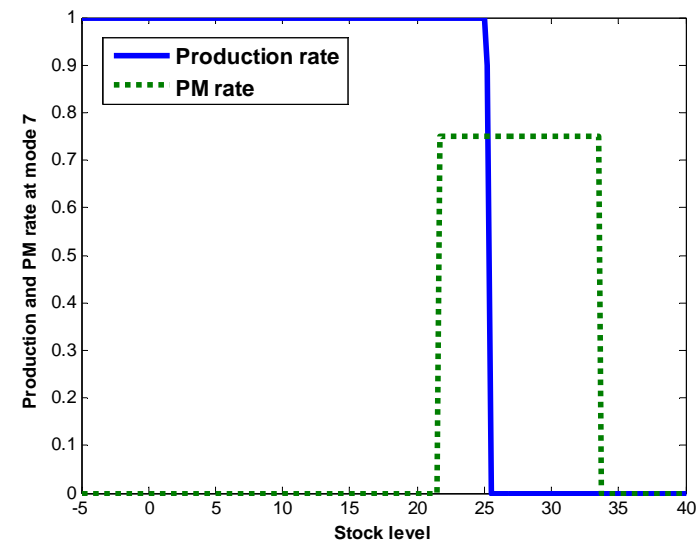

(d)

Figure 3 Production and PM rates at operational modes 3 and 7

From Figures 2 and 3, it follows that the production policy divides the plan $(x, \alpha)$ into three mutually exclusive regions in which the production rate is set to $u_{\max }, d$ and 0 , respectively. Furthermore, the maintenance policy also divides the plan $(x, \alpha)$ into three regions, such that the PM rate is alternatively $0, \omega_{\max }$ and 0 . If $Z_{1}^{\alpha}$ and $Z_{2}^{\alpha}$ denote the regions' limits for the production and the maintenance policies, respectively, this gives rise to the following machine modedependent control policy $(u(\cdot), \omega(\cdot))$ :

$$
\begin{gathered}
u(x, \alpha)=\left\{\begin{array}{ll}
u_{\max } & \text { if } x(\cdot)<Z_{1}^{\alpha} \\
d & \text { if } x(\cdot)=Z_{1}^{\alpha} \\
0 & \text { otherwise }
\end{array}, \omega(x, \alpha)= \begin{cases}\omega_{\max } & \text { if } x(\cdot) \geq Z_{2}^{\alpha} \\
0 & \text { otherwise }\end{cases} \right. \\
Z_{2}^{\alpha} \leq Z_{1}^{\alpha}, \alpha=1,2, \ldots, N-1, N
\end{gathered}
$$

As can be seen in Figure 3, $\left(Z_{1}^{3}, Z_{2}^{3}\right)=(4,3.75)$ and $\left(Z_{1}^{7}, Z_{2}^{7}\right)=(7,6)$ in Figures 3(a) and 3(c), whereas $\left(Z_{1}^{3}, Z_{2}^{3}\right)=(20,16)$ and $\left(Z_{1}^{7}, Z_{2}^{7}\right)=(25,22)$ in Figures $3(\mathrm{~b})$ and $3(\mathrm{~d})$. These results thus 
highlight both the fact that $Z_{1}^{\alpha}$ and $Z_{2}^{\alpha}$ increase when the degree of degradation increases, just as was observed in (Hu et al. 1995), and that $Z_{1}^{\alpha}$ and $Z_{2}^{\alpha}$ can not be deemed to be different in this example. If PM is scheduled at fixed periods $T_{\alpha}$ at mode $\alpha$, the control policy could be defined by three parameters $\left(T_{\alpha}, Z_{1}^{\alpha}, Z_{2}^{\alpha}\right)$, or by two parameters $\left(T_{\alpha}, Z_{1}^{\alpha}\right)$, if $Z_{1}^{\alpha} \cong Z_{2}^{\alpha}$.

Let us define a binary variable $\Omega($.) from equation (6) as follows:

$$
\Omega(x, \alpha)= \begin{cases}1 & \text { if } \omega(x, \alpha)=\omega_{\max } \\ 0 & \text { otherwise }\end{cases}
$$

At each $k T_{\alpha}, k=1,2,3 \ldots, \mathrm{PM}$ is either executed $(\Omega(x, \alpha)=1)$ or skipped $(\Omega(x, \alpha)=0)$. Using average values of these parameters (steady state), and for an infinite number of modes, the control policy could be approximated by a combination of a modified BRP (for the maintenance part) and an HPP (for the production/inventory part), and may thus be defined either by $\left(T, Z_{1}, Z_{2}\right)$ or $\left(T, Z_{1}\right)$, such that:

- PM strategy: PM scheduled at fixed time periods $k \cdot T, k=1,2,3 \ldots$ and executed if $x(\cdot) \geq Z_{2}\left(\operatorname{resp} . Z_{1}\right)$ and $\xi=1$ (i.e., $\Omega(x, \alpha)=1$ );

- Inventory policy: $u(\cdot)=\left\{\begin{array}{ll}u_{\max } & \text { if } x(t)<Z_{1} \text { and } \xi=1 \\ d & \text { if } x(t)=Z_{1} \text { and } \xi=1 \text {; } \\ 0 & \text { otherwise }\end{array}\right.$.

with $Z_{2} \leq Z_{1}$

where $\xi$ takes value in $\{1,2,3\}$, such that $\xi=1$ when the machine is operational, $\xi=2$ when the machine is under $\mathrm{CM}$, and $\xi=3$ when the machine is under PM.

For the sake of convenience, the two- and three-parameter policies are hereinafter denoted HPBJ1 and HPBJ2 (i.e., for "hedging point and block-replacement policies with jumps"), respectively. As a point of comparison, the classical policy that combines HPP and BRP without skipping any PM (Ki Ling et al. 1997) will also be considered and denoted by HPB (i.e., for "hedging point and block-replacement policies"). Note that the HPB and the HPBJ1 policies represent special cases of the generalized structure HPBJ2, where $Z_{2}$ is equal to $Z_{1}$ for HPBJ1, whereas $Z_{2}$ has a very low value (i.e., such that PM is never skipped) for HPB.

The stock trajectory for several maintenance cycles $[k T,(k+1) T]$ under the proposed joint control policies is illustrated in Figure 4. Since the HPBJ1 and HPB policies are based on two parameters $\left(T, Z_{l}\right)$, they are both presented in Figure 4(a) in order to facilitate their comparison, whereas the stock trajectory under the HPBJ2 is depicted in Figure 4(b). Each maintenance cycle has the same duration, and begins with PM (if not skipped), and ends at the next scheduled PM with the possibility of one or more machine breakdowns. During a maintenance intervention, the production is stopped and the inventory level decreases at rate $-d$, which results in backlogs and incurs shortage costs when it drops below 0 . Once the system is operational, production resumes and the inventory level increases at rate $u_{\max }-d$ (build-up period). If the machine does not fail during the build-up period, the inventory level will reach $Z_{1}$ and remain there, with a production rate set at $d$ until the next maintenance event.

With our formulation, more than one failure may occur in the same maintenance cycle, and failures may occur during the build-up periods, unlike model formulations in other papers that 


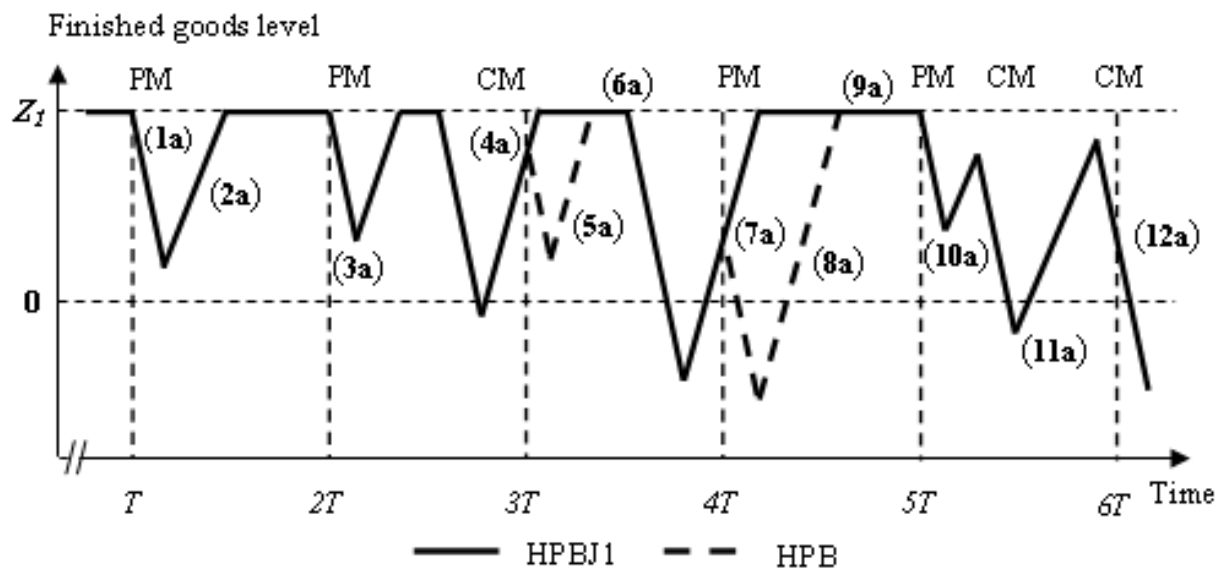

(a) HPBJ1 and HPB control policies

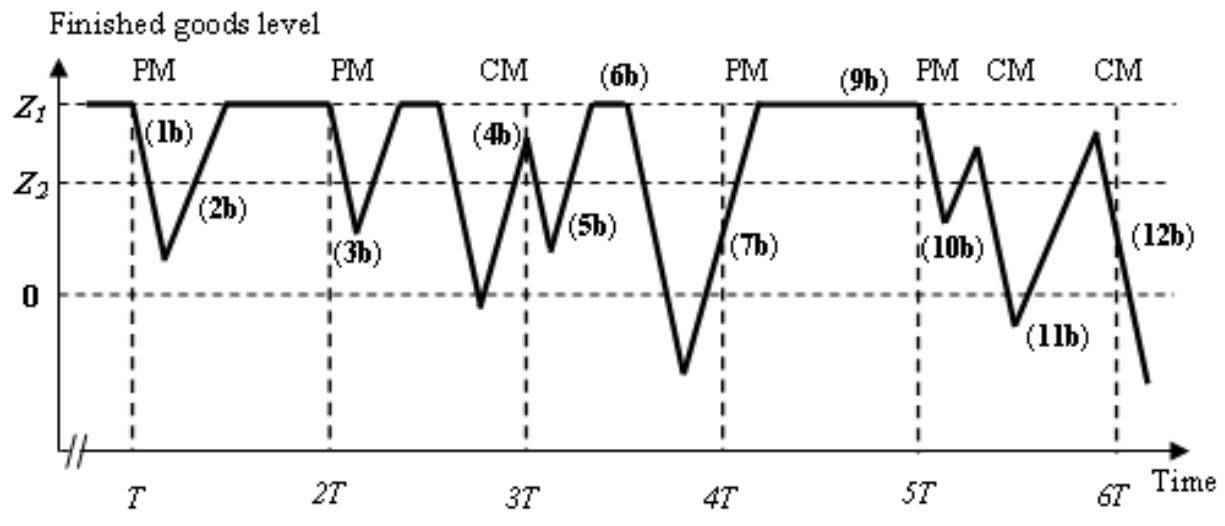

(b) HPBJ2 control policy

Figure 4 Stock trajectories under the proposed control policies

also look at PM and buffer stock control (Ki Ling et al. 1997; Chelbi and Ait-Kadi, 2004; Rezg,2004; Salameh et al. 2001). We also propose a relaxation of the assumption that the inventory level is periodic and null after PM (i.e., $x(k T)=0$ ), unlike in Chelbi and Ait-Kadi (2004), Salameh et al. (2001), Gharbi et al (2007), Rezg (2008).

The following maintenance cycles can be observed in Figure 4(a) and 4(b):

- $[T, 2 T]$ : Since $x(T)=Z_{1}$, PM is performed under the three policies ((1a) and (1b)). The system does not fail before the next scheduled PM, while the inventory increases $((2 \mathrm{a})$ and (2b)), and then is maintained at $Z_{l}$;

- $[2 T, 3 T]$ : Another PM is performed at time $2 T((3 a)$ and $(3 b))$. A failure occurs and triggers a CM action, which is completed before the end of the maintenance cycle. Production resumes not long before $3 T$, such that the inventory is not completely full at $3 T((4 a)$ and $(4 b))$. In Figure 4(a), $x(3 T)<Z_{1}$ for both the HPBJ1 and HPB policies, while $Z_{1}<x(3 T)<Z_{2}$ for the HPBJ2 policy (Figure 4(b));

- $[3 T, 4 T]$ : Under the HPBJ1 policy, PM is skipped, whereas PM is performed at $3 T$ under the HPBJ2 and HPB policies $((5 a)$ and $(5 b))$. In this scenario, the trajectory under the HPBJ1 and HPB policies splits into two different directions. Production resumes and the machine operates long enough for the inventory levels to reach the hedging 
point level ((6a) and (6b)), after which a failure occurs, and the inventory level at $4 T$ is below $Z_{2}$ for the HPBJ2 policy, and below $Z$ for the HPBJ 1 and HPB policies;

- $[4 T, 5 T]$ : The HPBJ1 and HPBJ2 policies both skip the scheduled PM, whereas PM is performed at $4 T$ as planned under the HPB policy ((7a) and (7b)). In this case, the HPB policy leads to a fall in $x(t)$ below 0 and to the wastage of a practically new component $((8 \mathrm{a}))$. A breakdown does not occur until the next scheduled PM in 5T, and thus the inventory levels increase ( $(9 \mathrm{a})$ and $(9 \mathrm{~b}))$ and reach the maximum level $Z_{1}$;

- $[5 T, 6 T]: \mathrm{PM}$ is performed at the beginning of the maintenance cycle at $5 T$ under the three policies ((10a) and (10b)). The system is then subject to several failures that occur in fairly quick succession ((11a) and (11b)), and the inventory never reaches $Z_{1}$. The last failure occurs before $6 T$ and ends after $6 T$. As a result, PM is skipped under the three policies ((12a) and (12b)).

Berg and Epstein (1976), as well as Archibald and Dekker (1996), established that skipping periodic PM to avoid the wastage of relatively new equipment enhances the performances of the BRP. When combined with an inventory control policy, skipping PM if $x(t)<Z_{2}$ should also reduce the risk of shortage due to consecutive $\mathrm{CM}$ and PM interventions. Moreover, this condition is indirectly equivalent to allowing a certain time for the inventory to build up before any PM intervention, and thus can be thought of as a condition on the machine age. Consequently, the HPBJ1 and HPBJ2 policies are expected to be doubly cost-effective as compared to the HPB policy. When comparing the HPBJ1 policy with the HPBJ2 policy, it is important to note that PM is skipped more often with the HPBJ1 policy than with the HPBJ2 policy since $Z_{2}<Z_{1}$, and the higher $Z_{2}$ is, the more often PM is skipped.

From the preceding discussion, it follows that once the control parameters are known (i.e., $\left(T, Z_{1}, Z_{2}\right)$ or $\left.\left(T, Z_{1}\right)\right)$, the near-optimal policies are completely defined. The numerical methods provide the structure of a near-optimal control policy. However, implementation difficulties and irregularities in the boundary of the numerical results make the approximation of the control parameters challenging. Furthermore, the accuracy of the value function and of the related control parameters obtained with the numerical methods depends on how fine the grid step is (Kenné et al. 2003). A satisfactory approximation would be too time-consuming to be applicable at the operational level, which is one of our objectives. In the next section, we propose an alternative approach based on simulation in order to approximate the optimal control parameters and the associated cost. The simulation-based approach is also more flexible and better suited to thoroughly compare the control policies in a wide range of time and cost configurations.

\section{Estimation of the optimal control policy}

\subsection{Control approach}

In the previous sections, the problem was analytically presented, and the structure of the optimal policy was obtained through a numerical approach. This section introduces the framework of an overall resolution approach that could easily be applied at an operational level. This approach, which is based on Kenné and Gharbi (1999) and Gharbi and Kenné (2000), combines both analytical and simulation models, an experimental design, and a statistical analysis. This approach is applied to the control problem proposed in the previous sections, and is described through the following six steps: 
- Step 1: Machine $\left(u^{*}, \omega^{*}\right)=$ ?: This step describes the objective of the study, which is to find the optimal production and maintenance control variables $u($.$) and \omega($.$) that$ minimize the total incurred maintenance and inventory cost.

- Step 2: Control problem formulation and analytical approach: Through an analytical approach (Section 2), and using numerical methods (Section 3), the structure of the optimal control policy is derived. Such a control policy is parameterized by the factors, $\left(T, Z_{1}, Z_{2}\right)$ or $\left(T, Z_{1}\right)$, and is taken as the input of the simulation model.

- Step 3: Simulation model: The simulation model evaluates the behaviour and the performance of the manufacturing system (i.e., the overall cost) for given values of the factors. Readers are referred to Section 4.2 for a detailed description of this simulation model.

- Step 4: Experimental design: The experimental design determines how the input factors should be varied using a minimal set of simulation runs. In order to obtain the effects of the main factors and their interactions on the incurred cost, a Pareto analysis is conducted on the data collected by simulation.

- Step 5: Response surface methodology: A regression analysis of significant factors and their interactions, in conjunction with a response surface methodology, provides the relationship between the cost and the input factors, and an estimation of their optimal values, called $\left(T^{*}, Z_{1}^{*}, Z_{2}^{*}\right)$ or $\left(T^{*}, Z_{1}{ }^{*}\right)$.

- Step 6: Selection of the best joint control policy: The three near-optimal control policies are then analyzed and compared for a wide range of cost and time parameters, from which we deduce the best control policy.

\subsection{Simulation model}

A simulation model was developed for each proposed control policy using the Visual SLAM simulation language with $\mathrm{C}$ sub-routines, and then executed through the AWESIM software application (Pritsker and O'Reilly, 1999). This model consists of several networks and user routines, each of which describes a specific task or event in the system (demand, failure and maintenance times, control policy, states of the system, threshold crossing of inventory variables..., etc.). We adopt a block diagram representation of the maintenance strategy and of the inventory control policy to facilitate understanding, as depicted in Figure 5 and Figure 6, respectively. The simulation model is detailed below.

State equations (C language insert)

The state equation (1) is defined as a $\mathrm{C}$ language insert. It describes the evolution of the inventory level $x(t)$ as a function of the demand rate and of the production rate, set by the control policy.

\section{HPP block-diagram}

In Figure 5, a detect node raises a signal whenever the hedging point $Z_{1}$ is crossed by $x(t)$ (SIGNAL block). Considering the state of the system, $(x, \xi)$, the production rate, $u($.$) , is then set$ according to the HPP. 


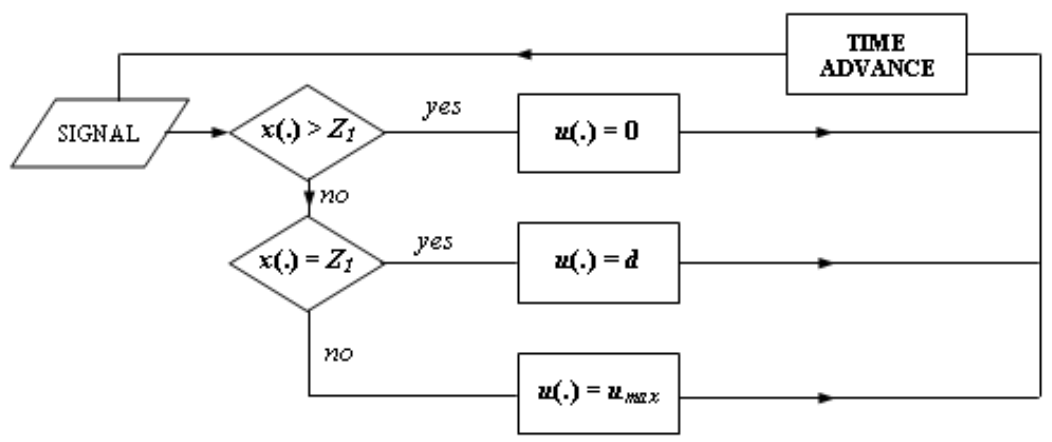

Figure 5 The HPP block-diagram of the simulation model

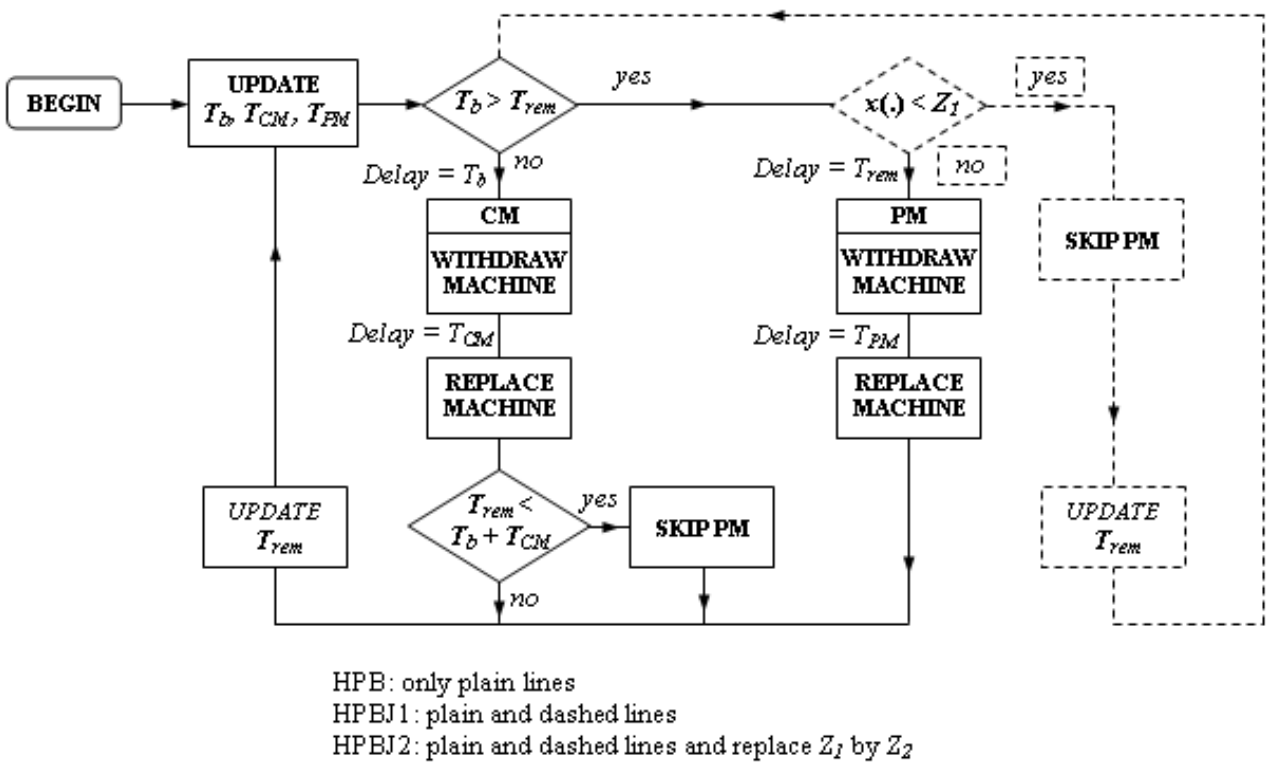

Figure 6 The maintenance control block-diagram of the simulation model

\section{Maintenance policy block-diagram}

The maintenance policies under the HPB, HPBJ1 and HPBJ2 policies are illustrated in Figure 6. The block diagram provides the system state changes (discrete events) and involves an additional variable, $T_{\text {rem }}$, which denotes the remaining time before the next scheduled PM. After each maintenance action (PM or CM), the "UPDATE $T_{b}, T_{C M}, T_{P M}$ " block samples the time to fail and the $\mathrm{CM}$ and PM durations from their respective probability distributions. Under the HPB policy, the maintenance policy is a simple BRP, which entails that the machine is instantaneously withdrawn and repaired during a delay, $T_{C M}(\mathrm{CM})$, when a failure occurs before the next scheduled PM (if $T_{b}<T_{\text {rem }}$ ), and periodically maintained with a delay, $T_{P M}$, every $T$ units of time (if $T_{b}>T_{\text {rem }}$ ). A block test checks the proposition " $T_{r e m}<T_{b}+T_{C M}$ ", which means that "a failure begins before and ends after the scheduled PM". If the proposition is true, the scheduled PM is skipped and the scheduled PM considered in $T_{\text {rem }}$ would be the next PM, $T$ units of time later. The "UPDATE $T_{\text {rem }}$ " block calculates the new value of $T_{\text {rem }}$ after a maintenance action or if PM is skipped. Under the HPBJ2 policy (respectively, HPBJ1), an additional branch is added to the 
block diagram in order to test whether the inventory level is below $Z_{2}$ (respectively, $Z_{1}$ ), and thus whether PM should be skipped. After any stoppage and resumption of production, the production block diagram updates the inventory level and the incurred cost.

\section{Production block}

The production rate, set by the HPP block, and the machine state, set by the maintenance strategy block, are brought together. The inventory level and the overall cost are updated according to equations (1) and (3) after the duration of a time step, which depends on discrete event scheduling (failures and maintenances), continuous threshold crossing events and time step specifications. For more details, the reader is referred to Gharbi and Kenné (2000). Once the simulation time reaches the predefined simulation horizon, the simulation run is stopped and the incurred cost for the given control parameters is obtained.

\subsection{Statistical analysis}

For each joint maintenance and inventory policy, two or three independent variables $\left(\left(T, Z_{1}\right)\right.$ for the HPB and HPBJ1 policies, $\left(T, Z_{1}, Z_{2}\right)$ for the HPBJ2 policy) and one dependent variable (the overall cost) are considered in the data collection. Assuming that the value function in equation (4) is convex, a second-order model is considered to fit the cost function. Complete $3^{2}$ (for the HPB and HPBJ policies) and $3^{3}$ (for the HPBJ2 policy) experimental designs are selected. Each combination of factors is replicated four times, requiring $36\left(3^{2} \mathrm{x} 4\right)$ and $108\left(3^{3} \mathrm{x}\right.$ 4) simulations runs, respectively.

First, a multifactorial analysis of variance (ANOVA) or a Pareto analysis is performed using a statistical software application (STATISTICA) in order to quantify the effects of the factors, their interactions and their quadratic effects on the incurred cost. Non-significant factors are eliminated with a level of significance set at 0.05 . This analysis also provides the proportion of the observed variability explained by the model and denoted by the R-squared adjusted value.

A response surface methodology is subsequently carried out in order to optimize the response (i.e., the incurred cost) as a function of the significant variables. We assume that there exists a continuous function $\Psi$, called the response surface, which provides the value of the cost corresponding to any given combination of $T$ and $Z_{1}$ (respectively, $T, Z_{1}$ and $Z_{2}$ ). The following functions are considered:

$$
\begin{gathered}
\psi_{H P B}(T, Z)=\alpha_{0}+\alpha_{1} \cdot T+a_{2} \cdot Z+\alpha_{11} \cdot T^{2}+\alpha_{22} \cdot Z^{2}+\alpha_{12} \cdot T \cdot Z+\varepsilon \\
\begin{aligned}
\psi_{H P B J}(T, Z)=\beta_{0} & +\beta_{1} \cdot T+\beta_{2} \cdot Z+\beta_{11} \cdot T^{2}+\beta_{22} \cdot Z^{2}+\beta_{12} \cdot T \cdot Z+\varepsilon \\
\psi_{H P B J 2}\left(T, Z_{1}, Z_{2}\right) & =\gamma_{0}+\gamma_{1} \cdot T+\gamma_{2} \cdot Z_{1}+\gamma_{3} \cdot Z_{2}+\gamma_{11} \cdot T^{2}+\gamma_{22} \cdot Z_{1}^{2}+\gamma_{33} \cdot Z_{2}{ }^{2} \\
& +\gamma_{12} \cdot T \cdot Z_{1}+\gamma_{13} \cdot T \cdot Z_{2}+\gamma_{23} \cdot Z_{1} \cdot Z_{2}+\varepsilon
\end{aligned}
\end{gathered}
$$

where $\left(\alpha_{0}, \alpha_{i}, \alpha_{i j}\right),\left(\beta_{0}, \beta_{i}, \beta_{i j}\right),\left(\gamma_{0}, \gamma_{i}, \gamma_{i j}\right),(i, j) \in\{1,2,3\}$ are unknown parameters and $\varepsilon$ is the residual error. Note that non-significant effects, third-order interactions and all other effects are ignored or are added to the error, $\varepsilon$.

Once the unknown parameters are estimated, the corresponding response surfaces are computed to give the coordinates of the optimal points for each control policy. For more details on the statistical analysis, readers are referred to Montgomery (2005). 


\section{Numerical example}

The determination of the optimal control parameters for the three policies is detailed in a step-bystep manner with a numerical example using the data parameters presented in Table 1 (basic case). The manufacturing system considered here is designed to address demand with a maximum production rate $20 \%$ higher than the nominal demand rate. The stochastic variables that describe the failure and maintenance times follow lognormal distributions (the values within parentheses indicate the mean and the standard deviations). Note that any probability distributions can be implemented in the simulation model. CM and PM differ in their costs and their durations, such that CM is twice as long and costs three times as much as PM. Backlogs are penalized by a cost ten times as high as the holding cost.

Table 1 Data parameters of the basic case

\begin{tabular}{ccccccccc}
\hline$u_{\max }$ & $D$ & $c_{1}$ & $c_{2}$ & $c^{+}$ & $c^{-}$ & $T_{b}$ & $T_{C M}$ & $T_{P M}$ \\
24 & 20 & 7500 & 2500 & 0.1 & 1 & Lognormal $(200,100)$ & Lognormal (20,2) & Lognormal (10,1) \\
\hline
\end{tabular}

A new variable is introduced, $z_{2}\left(0 \leq z_{2} \leq 1\right.$ and $\left.Z_{2}=z_{2} \cdot Z_{1}\right)$, in order to ensure that $Z_{2} \leq Z_{1}$. For each replication, the simulation horizon is set to $5,000,000$ units of time to ensure that the steady state is achieved.

The quantified effects of the main factors, interactions and quadratic effects (with standardized factors), and the R-squared adjusted value for each control policy, are shown in Figure 7. This figure indicates that all factors are significant. The variability of the simulation model for the HPB, HPBJ1 and HBBJ2 policies (i.e., the R-squared adjusted values) explains 98.98\%, 98.29\% and $97.89 \%$ of the variability observed in the overall costs, respectively. It also confirms that the overall costs are convex and can be fitted by second-order models. A residual analysis was used to verify the adequacy of these models.
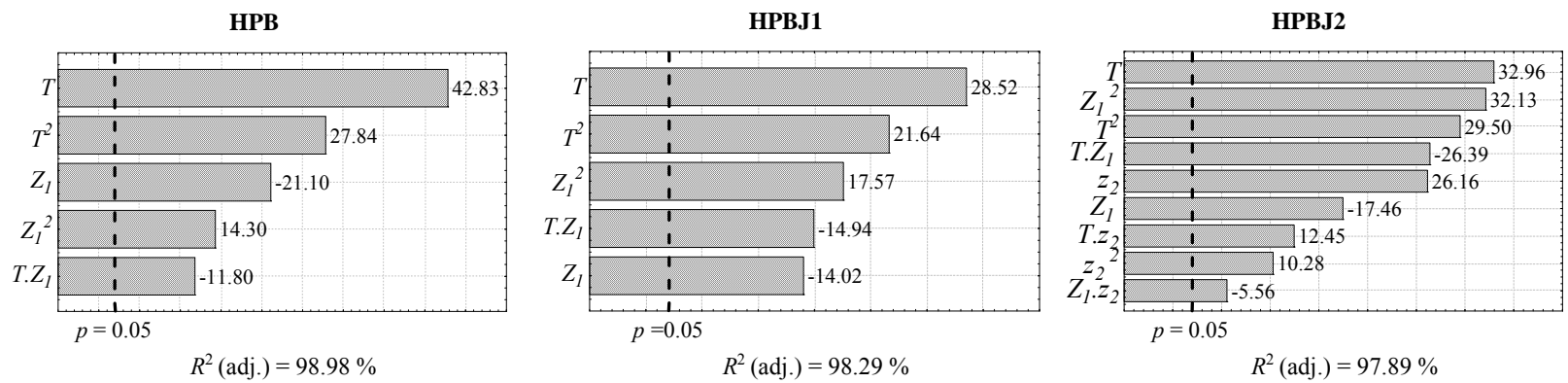

Figure 7 Pareto chart under the three policies

The corresponding second-order models are given by:

$\psi_{H P B}=51.302+2.8709 \cdot T+4.0661 \cdot T^{2}-1.4201 \cdot Z_{1}+2.5516 \cdot Z_{1}^{2}-1.2540 \cdot T \cdot Z_{1}+\varepsilon$ 


$$
\begin{aligned}
\psi_{H P B J}= & 48.346+0.0642 \cdot T+1.9888 \cdot T^{2}-0.18723 \cdot Z_{1}+2.3755 \cdot Z_{1}^{2}-0.8240 \cdot T \cdot Z_{1}+\varepsilon \\
\psi_{H P B J 2}= & 47.335-0.3714 \cdot T+2.0024 \cdot T^{2}+0.01309 \cdot Z_{1}+2.4398 \cdot Z_{1}^{2}+0.4567 \cdot z_{2} \\
& +0.5031 \cdot z_{2}{ }^{2}-0.8203 \cdot T \cdot Z_{1}+0.3402 \cdot T \cdot z_{2}-0.1693 \cdot Z_{1} \cdot z_{2}+\varepsilon
\end{aligned}
$$

The projection of the cost response surfaces onto two-dimensional planes is presented in Figure 8 for the HPBJ2 policy. The minimum total cost is, $J_{H P B J 2} *=\$ 47.20$, and is located at $T^{*}=92.71, Z_{1}^{*}=200.14$ and $z_{2}^{*}=0.25\left(Z_{2}^{*}=50.11\right)$. These values represent the best parameters of the near-optimal control policy, which should be applied to control both the maintenance and inventory of the manufacturing system. Similarly, the control parameters that minimize the cost response surface of the HBP and HPBJ1 policies are computed.
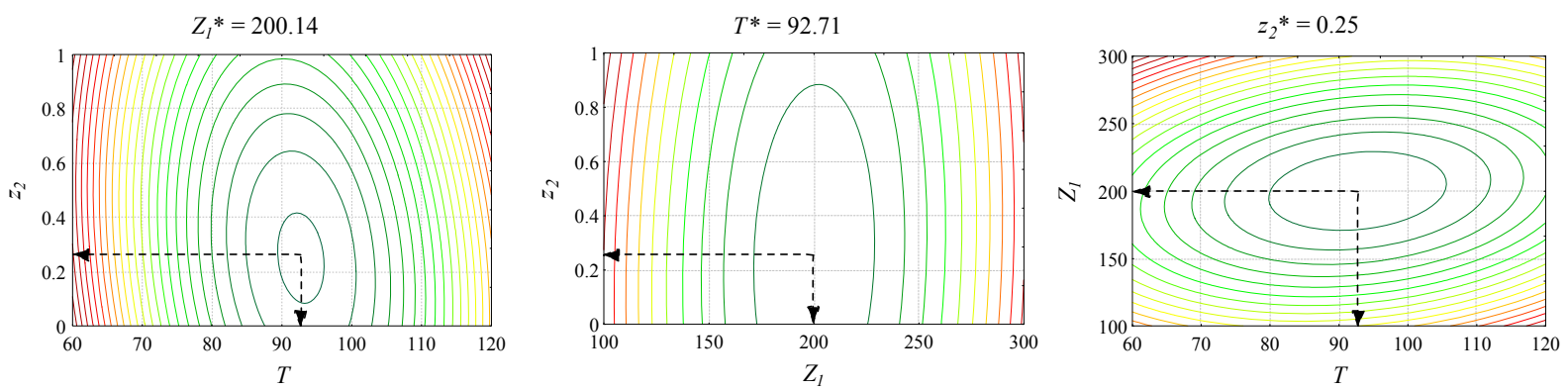

Figure 8 Cost response surfaces under the HPBJ2 policy

\subsection{Comparison of the performances of the control policies}

Table 2 indicates several relevant measures that complete the measure of the total cost. They were obtained with the values of the control parameters presented in the last section.

Table 2 Complementary performance indices measured by simulation

\begin{tabular}{lccccccccccccc}
\hline Policy & $T^{*}$ & $Z_{1}^{*}$ & $z_{2}{ }^{*}$ & $\bar{X}^{+}$ & $\bar{X}^{-}$ & $\begin{array}{c}\text { No. of } \\
\text { CM }\end{array}$ & $\begin{array}{c}\text { No. of } \\
\text { PM }\end{array}$ & $\begin{array}{c}\text { No. of } \\
\text { skipped } \\
\text { PM }\end{array}$ & $A(\%)$ & $L$ & $J^{i}$ & $J^{m}$ & $J^{*}$ \\
\hline HPBJ2 & 92.71 & 200.14 & 0.25 & 126.80 & 4.96 & 2941 & 50739 & 1104 & 88.61 & 82.60 & 17.42 & 29.78 & 47.20 \\
HPBJ1 & 90.14 & 202.03 & 128.87 & 5.53 & 3671 & 49301 & 4038 & 88.67 & 83.70 & 18.22 & 30.12 & 48.34 \\
HPB & 83.55 & 209.95 & 128.78 & 6.10 & 1793 & 58382 & & 87.61 & 72.79 & 18.69 & 32.01 & 50.70 \\
\hline
\end{tabular}

where $\bar{X}^{+}$and $\bar{X}^{-}$denote the average positive and negative inventory levels, $\mathrm{A}$ is the average availability, $\mathrm{L}$ is the average machine lifetime, $J^{i}, J^{m}$ and $J^{*}$ are the incurred inventory, maintenance and total cost, respectively.

- Maintenance performance: Table 2 shows that the performances of the HPBJ1 and HPBJ2 policies in terms of availability, average lifetime and maintenance costs are almost the same. Both the HPBJ1 and HPBJ2 policies outperform the HPB policy with respect to maintenance performance, because the HPB policy leads to the wastage of 
relatively new components. Relatively few PM are skipped under the HPBJ1 and HPBJ2 policies, as compared to the amount of PM realized. However the availability and the average lifetime of the machine are enhanced.

- Inventory performance: Since skipping PM enables the spacing of maintenance actions and prevents a significant decrease in the inventory level due to consecutive inactive periods, the inventory costs observed under the HPBJ1 and HPBJ2 policies are better than those seen under the HPB policy. The optimal value of $Z_{l}$ and $T$ required to avoid failures and backlogs are respectively higher and smaller with the HPB policy. In addition, it can be seen that the simplification of $Z_{2}=Z_{1}$ with the HPBJ1 policy leads to more skipped PM, but also to more failures, more backlogs and a higher inventory cost than in the case of $Z_{2} \neq Z_{1}$, which is proposed in the HPBJ2 policy.

The main conclusion to be drawn from this analysis is that the HPBJ2 policy provides a better approximation of the real optimal policy than the HPBJ1 policy in this example, since $Z_{1}{ }^{*}$ and $Z_{2}{ }^{*}$ are different, and the incurred cost is $2.82 \%$ better than that under the HPBJ1 policy. The cost difference is derived from both the inventory and the maintenance costs.

When studying the availability of the system through an ANOVA analysis, the availability under the HPB and HPBJ1 policies is shown to be a function of $T$, and is independent of $Z_{1}$. Concerning the HPBJ2 policy, an ANOVA analysis reveals that $z_{2}$ is also significant. Though $z_{2}$ does affect the number of PM breaks, its effects on the availability are however small as compared to those of $T$. Figure 9 shows availability as a function of $T$ under the three proposed policies.

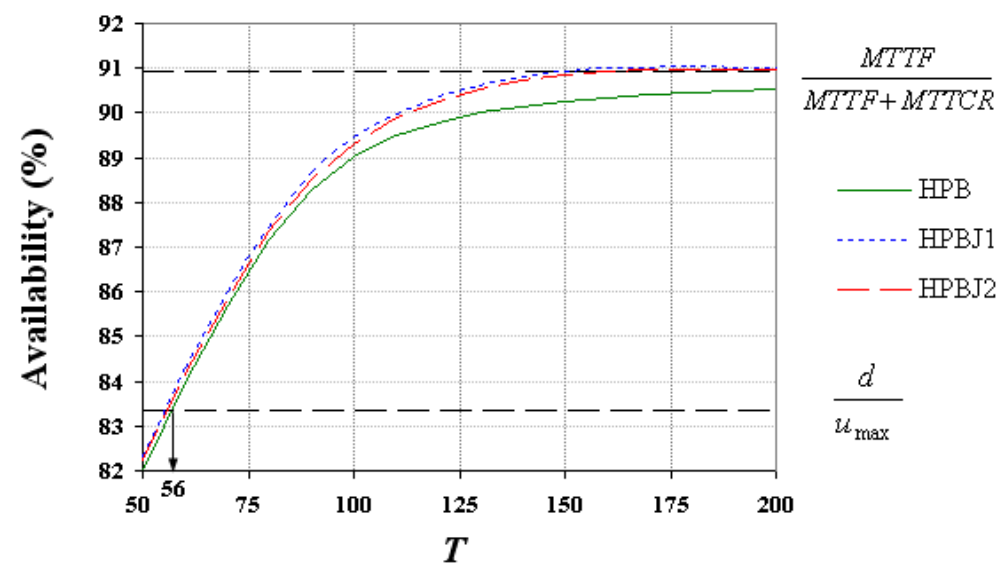

Figure 9 Availability as a function of $\boldsymbol{T}$ under the three policies

Figure 9 highlights the fact that the availability of the system increases when $T$ increases or when more PM are skipped (i.e., if PM are performed less often). Consequently, the availability under the HPBJ2 policy is slightly lower than that for the HPBJ1 policy, and better than that for the HPB policy for a given $T$. Figure 9 also shows the feasibility limit of the system $\left(A>d / u_{\max }\right.$ ), which is equivalent to imposing a minimal value for $T$ (i.e., $T>56$ for the HPB policy). However, if $T$ tends to infinity, the availabilities tend to the same asymptotical limit (MTTF / $(M T T F+M T T C R)$, where MTTF is the mean time to failure), which is the availability under a failure replacement strategy. 


\section{Sensitivity analysis and comparative study}

A sensitivity analysis of the control policies is conducted with respect to cost parameters. Its objective is to compare the incurred costs of the control policies for different cost combinations derived from the basic case and presented in Table 3. The results are summarized in Table 4, where the consistency between the variation of each cost parameter and the optimal costs and control parameters is highlighted.

Table 3 Combination of cost parameters of the sensitivity analysis

\begin{tabular}{ccccc}
\hline Case & $c_{1}$ & $c_{2}$ & $c^{+}$ & $c^{-}$ \\
\hline 1 & $\mathbf{7 5 0 0}$ & $\mathbf{2 5 0 0}$ & $\mathbf{0 . 1}$ & $\mathbf{1}$ \\
2 & $\mathbf{5 0 0 0}$ & 2500 & 0.1 & 1 \\
3 & $\mathbf{1 0 0 0 0}$ & 2500 & 0.1 & 1 \\
4 & 7500 & $\mathbf{2 0 0 0}$ & 0.1 & 1 \\
5 & 7500 & $\mathbf{3 0 0 0}$ & 0.1 & 1 \\
6 & 7500 & 2500 & $\mathbf{0 . 0 5}$ & 1 \\
7 & 7500 & 2500 & $\mathbf{0 . 1 5}$ & 1 \\
8 & 7500 & 2500 & 0.1 & $\mathbf{0 . 5}$ \\
9 & 7500 & 2500 & 0.1 & $\mathbf{1 . 5}$ \\
\hline
\end{tabular}

Table 4 Sensitivity analysis for different cost parameters

\begin{tabular}{|c|ccc|ccc|c|ccc|cc|}
\cline { 2 - 13 } \multicolumn{1}{c|}{} & \multicolumn{4}{c|}{$T$} & \multicolumn{3}{c|}{$Z_{1}$} & $Z_{2}$ & \multicolumn{3}{c|}{ Total cost } & \multicolumn{2}{c|}{ Costs difference (\%) } \\
\hline \multirow{2}{*}{ Case } & HPB & HPBJ1 & HPBJ2 & HPB & HPBJ1 & HPBJ2 & HPBJ2 & HPB & HPBJ1 & HPBJ2 & HPBJ1 & HPBJ2 \\
/ HPB & / HPBJ1 \\
\hline 1 & 83.55 & 90.14 & 92.71 & 209.95 & 202.03 & 200.14 & 5.01 & 50.70 & 48.34 & 47.20 & -4.65 & -2.43 \\
2 & 86.04 & 96.65 & 99.44 & 211.48 & 204.85 & 202.62 & 4.72 & 49.73 & 46.36 & 45.58 & -6.77 & -1.72 \\
3 & 81.30 & 85.32 & 87.40 & 208.57 & 199.94 & 198.17 & 4.89 & 51.56 & 50.02 & 48.47 & -2.98 & -3.21 \\
4 & 79.13 & 82.74 & 84.39 & 207.24 & 198.82 & 196.91 & 5.23 & 44.67 & 43.16 & 41.79 & -3.37 & -3.27 \\
5 & 87.36 & 96.62 & 99.50 & 212.29 & 204.84 & 202.59 & 3.93 & 56.40 & 53.07 & 52.07 & -5.91 & -1.92 \\
6 & 86.29 & 93.90 & 96.50 & 234.96 & 228.72 & 226.06 & 6.31 & 43.69 & 41.25 & 40.22 & -5.58 & -2.56 \\
7 & 80.36 & 85.58 & 88.03 & 186.69 & 177.18 & 176.03 & 3.94 & 56.52 & 54.18 & 52.94 & -4.14 & -2.33 \\
8 & 89.01 & 96.24 & 99.82 & 172.48 & 160.83 & 159.69 & 0.38 & 46.62 & 44.54 & 43.68 & -4.47 & -1.96 \\
9 & 80.71 & 86.12 & 88.04 & 223.52 & 216.73 & 214.33 & 6.69 & 53.19 & 50.54 & 49.05 & -4.98 & -3.04 \\
\hline
\end{tabular}

Both the HPBJ1 and HPBJ2 policies outperform the HPB policy (the total cost under the HPBJ1 policy is $2.98 \%$ to $6.77 \%$ better than under the HPB policy). The HPBJ2 policy outperforms the HPBJ1 policy ( $1.72 \%$ to $3.27 \%$ better), and the PM threshold $Z_{2}$ * is significantly lower than the hedging point $Z_{1} *$ in all cases. The optimal parameters evolve in the same directions, and with the same magnitude, in response to any variations in cost parameters. Furthermore, the optimal values of the hedging points, $Z_{1}{ }^{*}$, under the HPB policy are higher than those observed under the HPBJ1 and HPBJ2 policies, which are almost equal (since their maintenance performances are almost the same). The reason for this is that the system tends to protect itself against backlogs due to consecutive CM and PM. The variations in the cost parameters illustrated in Table 4 are examined and are compared to the basic case as follows: 
- Variation in the CM cost, $c_{1}$ (cases 2 and 3): When the CM cost increases, the interval between scheduled PM, $T^{*}$ (and the PM threshold $Z_{2}{ }^{*}$ ) decrease to give preference to PM interventions and to avoid breakdowns. Consequently, the risk of breakdown and shortage decreases (i.e., MTTPM $<M T T C R$ ), and inventory control requires a lower hedging point, $Z_{1}^{*}$.

- Variation in the PM cost, $c_{2}$ (cases 4 and 5): The variation in the PM cost has the opposite effect when compared to that of the $\mathrm{CM}$ cost because both cost variations influence the optimal balance between PM and CM in different directions.

- Variation in the inventory cost, $c^{+}$(cases 6 and 7): When the inventory cost increases, lower average inventory must be kept on hand, and thus the hedging point, $Z_{1}$ *, decreases for the three control policies. To avoid the risk of shortage when the average inventory level decreases, the system reacts by reducing the period between scheduled $\mathrm{PM}, T^{*}$, and the PM threshold, $Z_{2}{ }^{*}$.

- Variation in the backlog cost, $c^{-}$(cases 8 and 9): When the backlog cost is higher, higher stock levels must be kept and long inactive periods due to breakdowns avoided. To protect the system from shortages, the hedging point, $Z_{1}^{*}$, is increased for the three control policies, and PM are performed more frequently.

To confirm the cost differences observed in the sensitivity analysis, a Student's t-test is conducted on the cost difference between the HPB and HPBJ1 policies and between the HPBJ1 and HPBJ2 policies with the parameter data shown in Table 3. The confidence interval of $J_{H P B}^{*}-J_{H P B J 1}^{*}$ is given by:

$$
\bar{J}_{H P B}^{*}-\bar{J}_{H P B J 1}^{*} \pm t_{\frac{\alpha}{2}, n-1} \cdot \operatorname{se} e\left(\bar{J}_{H P B}^{*}-\bar{J}_{H P B J 1}^{*}\right)
$$

where $t_{\frac{\alpha}{2}, n-1}$ is the student coefficient function of $n$ and $\alpha$, where $n$ denotes the number of replications (set at 20), and (1- $\alpha$ ) the confidence level (set at 95\%);

$$
\text { s.e }\left(\bar{J}_{H P B}^{*}-\bar{J}_{H P B J 1}^{*}\right)=\frac{S_{D}}{\sqrt{n}} \text { with } S_{D}=\left(\sum_{i=1}^{n}\left(J_{H P B}^{i *}-J_{H P B J 1}^{i *}\right)^{2}-n \cdot\left(\bar{J}_{H P B}^{*}-\bar{J}_{H P B J 1}^{*}\right)^{2}\right)
$$

$\bar{J}_{H P B J 1}^{*}$ is the average optimal cost under the HPBJ1 policy: $\bar{J}_{H P B J 1}^{*}=\frac{1}{n} \cdot \sum_{i=1}^{n} J_{H P B J 1}^{i^{*}}$;

$\bar{J}_{H P B}^{*}$ is the average optimal cost under the HPB policy: $\bar{J}_{H P B}^{*}=\frac{1}{n} \cdot \sum_{i=1}^{n} J_{H P B}^{i^{*}}$;

The control policies are simulated with their optimal control parameters as inputs for each combination of cost parameters. The confidence intervals of the incurred cost differences $J_{H P B}^{*}-J_{H P B J 1}^{*}$ and $J_{H P B J 1}^{*}-J_{H P B J 2}^{*}$ are presented in Table 5 and Table 6.

Table 5 Cost difference confidence interval (95\%) for HPBJ1 and HPB policies

\begin{tabular}{cccccccccc}
\multicolumn{10}{c}{$J_{H P B}^{*}-J_{H P B J 1}^{*}$} \\
\hline Case & 1 & 2 & 3 & 4 & 5 & 6 & 7 & 8 & 9 \\
\hline Lower bound & 2.193 & 3.027 & 1.894 & 2.014 & 3.004 & 2.208 & 2.412 & 1.584 & 2.851 \\
Upper bound & 2.356 & 3.189 & 2.041 & 2.151 & 3.170 & 2.346 & 2.566 & 1.722 & 3.023 \\
\hline
\end{tabular}


Table 6 Cost difference confidence interval (95\%) for HPBJ2 and HPBJ1 policies

\begin{tabular}{cccccccccc}
\multicolumn{10}{c}{$J_{\text {HPBJ1 }}^{*}-J_{H P B J 2}^{*}$} \\
\hline Case & 1 & 2 & 3 & 4 & 5 & 6 & 7 & 8 & 9 \\
\hline Lower bound & 1.064 & 0.800 & 1.293 & 1.444 & 0.794 & 0.063 & 1.439 & 0.322 & 1.176 \\
Upper bound & 1.261 & 0.963 & 1.507 & 1.640 & 0.994 & 0.242 & 1.665 & 0.489 & 1.427 \\
\hline
\end{tabular}

Since the lower bound of the confidence intervals of $J_{H P B}^{*}-J_{H P B J 1}^{*}$ and $J_{H P B J 1}^{*}-J_{H P B J 2}^{*}$ is positive in all cases at a 95\% confidence level, it can be concluded that both the HPBJ1 and the HPBJ2 policies outperform the HPB policy for the case studied $\left(J_{H P B J 2}^{*}<J_{H P B J 1}^{*}<J_{H P B}^{*}\right)$. Furthermore, the HPBJ2 policy is as easy to manage in practice as the HPB policy, which is a combination of an HPP with a classical periodic PM strategy, and only requires that the inventory level be checked at the scheduled PM times.

\section{Effects of cost and time parameters on the selection of the best control policy}

\subsection{Effects of cost parameters on the control policies}

The previous section showed that the overall costs under the HPBJ1 and HPBJ2 policies are relatively equal. The objective of this section is to conduct a more thorough examination of the effect of each cost parameter, separately or in pairs, on the joint control policies. Since the HPB policy has been used in the literature to study the joint inventory and maintenance control policies (Ki Ling et al. 1997), the objective is to compare the relative cost differences between the HPBJ1 and HPBJ2 policies and the HPB policy (i.e., $\frac{J_{H P B J 1}^{*}-J_{H P B}^{*}}{J_{H P B}^{*}}$ and $\frac{J_{H P B J 2}^{*}-J_{H P B}^{*}}{J_{H P B}^{*}}$ ) for a wide range of cost configurations, and to verify how the proposed control policies evolve in relation to one another. The effects of the inventory and of the backlog costs, $c^{+}$and $c^{-}$, are depicted in Figures 10(a) and 10(b), respectively. Similarly, Figures 11(a) and 11(b) present the effects of the $\mathrm{CM}$ and of the PM costs $c_{1}$ and $c_{2}$ on the incurred costs. All other cost parameters remain constant at values given in the basic case.

Keeping in mind that skipping PM becomes less attractive when the holding cost increases (as indicated in Table 4), the cost differences with the HPB policy decrease (Figure 10(a)). Figure 10(b) shows that increasing $c^{-}$will penalize the HPB policy more and more, because it results in consecutive CM and PM periods, and thus in more shortages. Nevertheless, as more PM are skipped under the HPBJ1 policy than under the HPBJ2 policy, the HPBJ1 policy triggers more failures (refer to Table 2), and leads to more shortages. The HPBJ1 policy will become less and less profitable in comparison with the HPBJ2 policy. When $c^{-}=10$, the HPBJ2 policy and the HPBJ1 policy are nearly $25 \%$ and $12 \%$ better than the HPB policy, respectively. When $c^{-}=c^{+}=0.1$ (i.e., if backlogs are not penalized), skipping PM will only influence the wastage of relatively new components under the HPB policy, hence the HPBJ1 and HPBJ2 policies will be close in terms of cost and both slightly better than the HPB policy (nearly $3 \%$ better). 


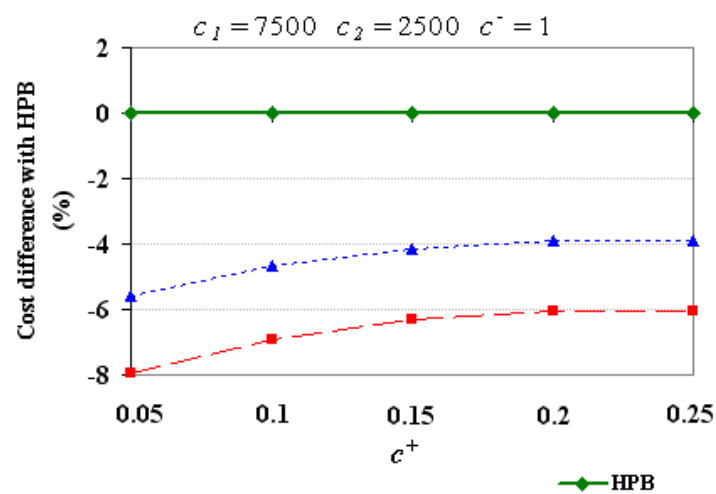

(a)

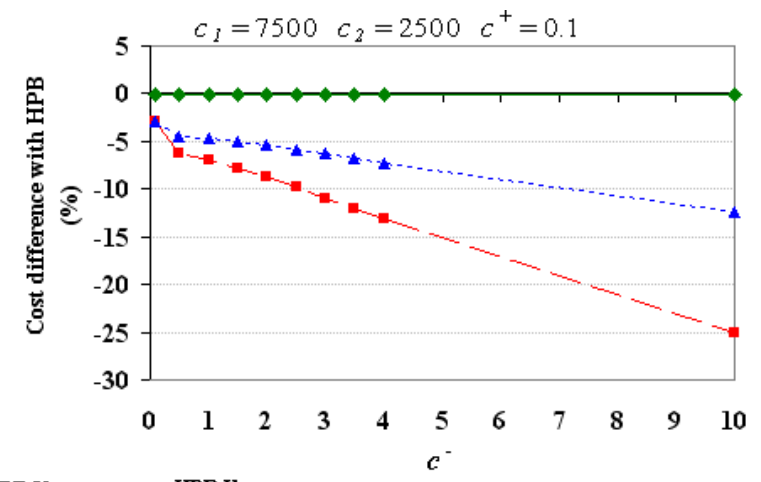

(b)

Figure 10 Cost comparison of the control policies with different holding and backlog costs

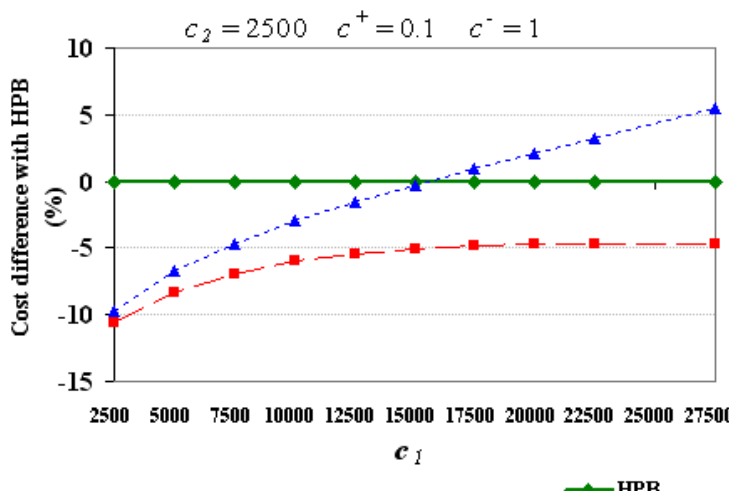

(a)

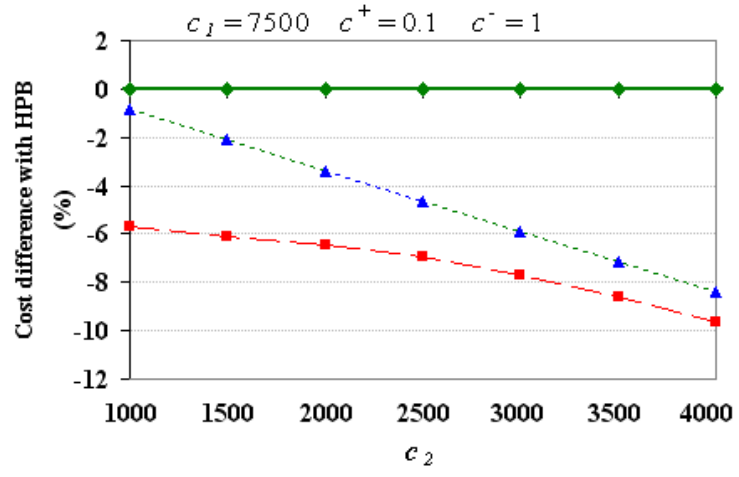

(b)

Figure 11 Cost comparison of the control policies with different CM and PM costs

Since the HPBJ1 and HPBJ2 policies lead to more failures than does the HPB policy, a significant increase in $\mathrm{CM}$ cost makes PM breaks less attractive, and may lead to situations where the HPBJ1 becomes less cost-effective than the HPB policy (Figure 11(a)). In that case, the incurred penalty costs from breakdowns (under the HPBJ1 policy) become greater than the benefits gained from avoiding the wastage of relatively new components (under the HPB policy). However, for large values of $c_{1}$, the HPBJ2 policy is still nearly $5 \%$ better in terms of cost than the HPB policy. If $c_{1}=c_{2}=2500$, breakdowns will only be penalized by MTTCR $>M T T P M$ and the HPBJ1 policy becomes almost as good as the HPBJ2 policy, and nearly $10 \%$ better than the HPB policy. Figure 11(b) confirms that the variation in PM costs has the opposite effect when compared with the variation in CM cost which was observed in Table 4.

Finally, Figure 12(a) and 12(b) illustrate the variations in the incurred costs when varying the relative weight of the inventory costs (i.e., backlog and holding costs) and the maintenance costs (i.e., CM and PM costs). If the relative weight of the inventory cost in the overall cost increases (respectively, if the weight of the maintenance cost decreases), the HPBJ2 and HPBJ1 policies are still superior to the HPB policy, which confirms that the concept of skipping PM is profitable. In the absence of the inventory costs, the HPBJ1 and HPBJ2 policies are equivalent, and skipping PM is motivated solely by the desire to avoid the wastage of relatively new components (Berg and Epstein, 1976; Archibald and Dekker, 1996). When the inventory costs increase, the influence of the backlog cost increases and the cost differences between the three joint control policies increases. 


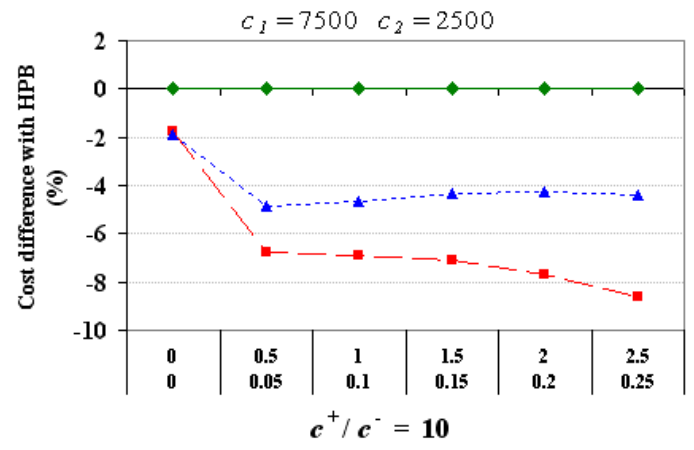

(a)

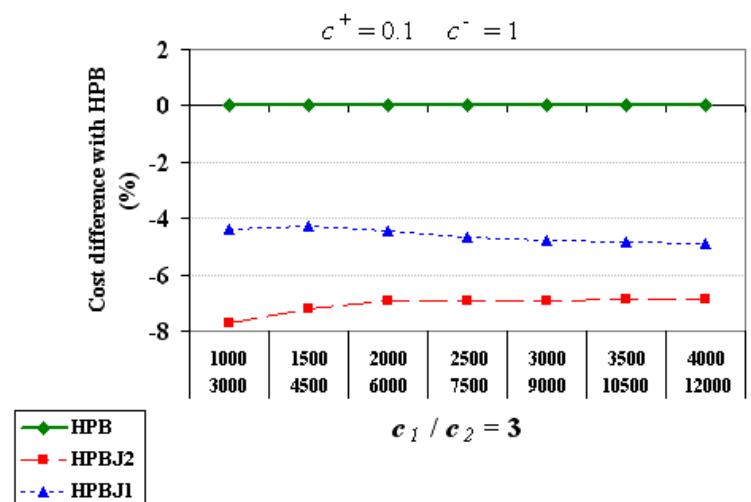

(b)

Figure 12 Cost comparison of the control policies with different inventory costs-to-maintenance costs ratio

The main conclusion is that the HPBJ2 policy constitutes a better approximation of the optimal control policy. It confirms that choosing to have two different inventory thresholds, $Z_{1}$ and $Z_{2}$, instead of only one, is more profitable. However, for lower values of the backlog, when $\mathrm{CM}$ and $\mathrm{PM}$ costs are relatively close, or when the relative weight of the inventory costs is lower, the overall costs of the HPBJ1 and HPBJ2 policies are similar.

\subsection{Effects of the CM duration on the control policies}

The influence of the mean and standard deviations of the time to perform a CM (MTTCR and $S D C$ ) and of the mean and standard deviations of the time to perform a PM (MTTPM and SDP) on cost differences are presented in Figures 13(a) and 13(b) and Figures 14(a) and 14(b), respectively. The cost parameters and all time parameters, except the considered time parameters, are set to their values defined in the basic case.

Figure 13(a) shows that when MTTCR decreases and tends to MTTPM $=10, \mathrm{CM}$ will only be penalized by the maintenance cost $\left(c_{1}>c_{2}\right)$. In this situation, the system allows more PM breaks to occur, and the HPBJ2 and HPBJ1 policies are equivalent $\left(Z_{2}=Z_{1}\right)$, and both better than the HPB policy. When MTTCR increases, the HPBJ1 policy becomes less attractive than the HPB and HPBJ2 policies since this policy causes the most machine breakdowns amongst the three policies. For large MTTCRs, $Z_{2}$ * takes lower values, and fewer scheduled PM are skipped, but just enough to enhance the performance compared to the HPB policy. In Figure 13(b), skipping PM is not necessary for lower values of MTTPM, and the HPB and HPBJ2 policies are equivalent and both are better than the HPBJ1 policy. If MTTPM increases and tends to MTTCR $=20$, the system adapts itself, avoiding most of the PMs under the three policies. As none of the PM interventions are skipped under the HPB policy, the best HPB policy will be a failure replacement maintenance policy ( $T^{*}$ tends towards infinity). Since the HPBJ1 and HPBJ2 policies selectively skip PM, both these policies become better than the HPB policies. Though the optimal value of $Z_{2}^{*}$ is high, it however differs from that of $Z_{1}{ }^{*}$, still leaving the HPBJ2 policy better than the HPBJ1 policy.

Figure 14(a) and 14(b) show that the control policies are affected by the variability of the maintenance durations, such that the more scattered the maintenance durations, the more attractive skipping PM becomes. It can also be noted that if the PM duration is highly variable, then $Z_{2}{ }^{*}$ tends to $Z_{1}$ * and the HPBJ and HPBJ2 policies become equivalent. 


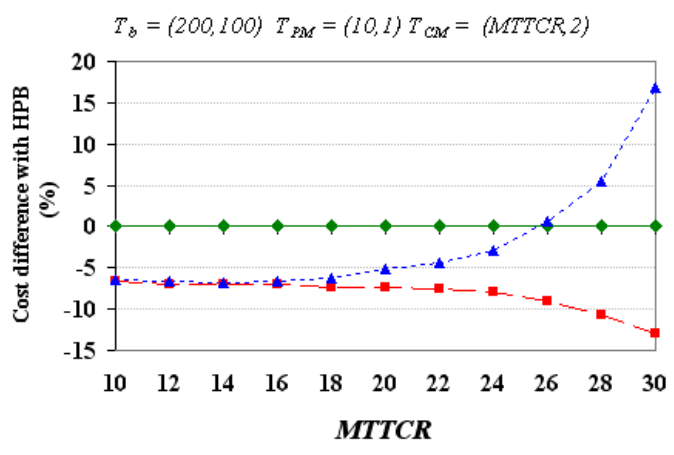

(a)

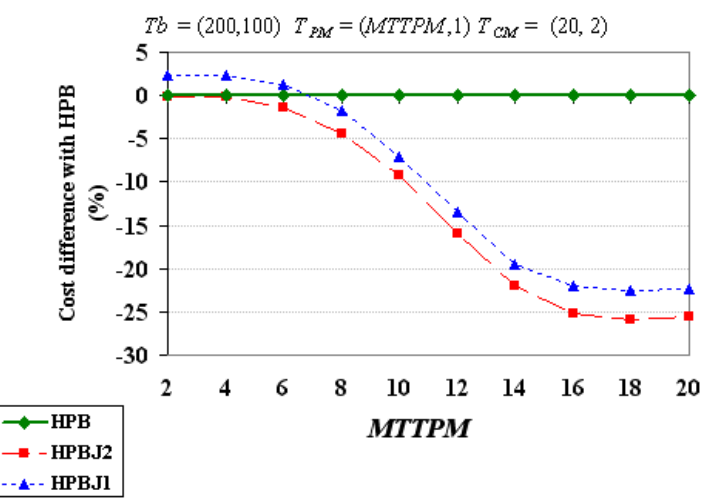

(b)

Figure 13 Cost comparison of the control policies with different mean times to perform CM and PM

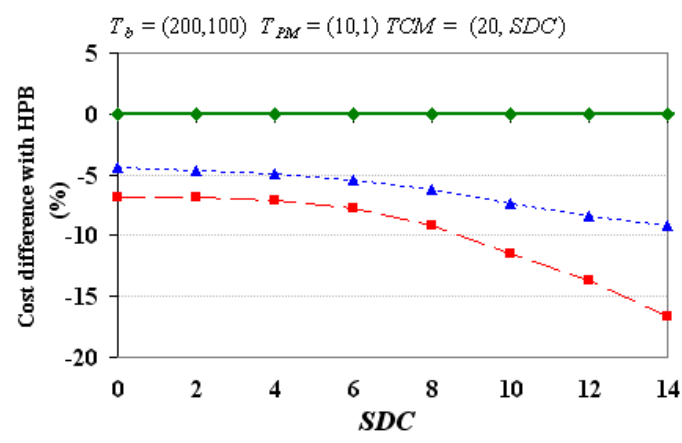

(a)

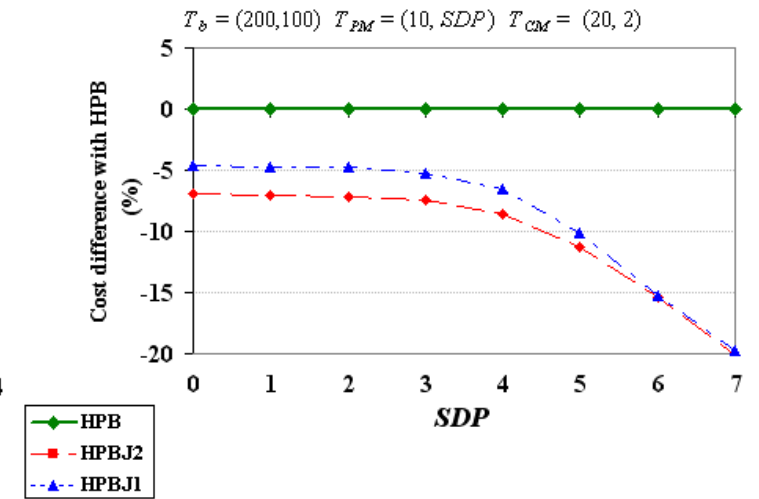

(b)

Figure 14 Cost comparison of the control policies with different standard deviations of the time to perform CM and PM

\section{Conclusion}

In this paper, the interactions between maintenance and production control in a manufacturing system are investigated in the case of non-negligible and random maintenance durations and with periodic preventive maintenance. To our knowledge, none of the inventory control policies with periodic PM proposed so far in the literature are optimal. These models are also limited by restrictive assumptions concerning the occurrence of breakdowns during stock build-up periods, loss of unmet demand and the periodicity of the inventory trajectory. We relax these restrictive assumptions and determine the approximated structure of the optimal joint maintenance and inventory control policy using numerical techniques. This policy consists of a classical HPP, to control the production rate, and a modified periodic PM strategy, in which the scheduled PM interventions are performed only if the inventory is at a sufficient level. This leads to a threeparameter joint control policy. A simplification of the joint control policy is also proposed, by considering that the inventory threshold that triggers the execution of PM and the hedging point are both at the same level. As a point of comparison, the classical control policy that combines HPP with a periodic PM strategy without skipping any PM is also considered. The optimal values of the corresponding control parameters for the three control policies are then determined through a simulation-based experimental approach. 
This approach is applied on a wide range of time and cost parameter configurations in order to evaluate their effects on the system under the proposed control policies. The main conclusion is that the joint control policy with three parameters is better than the simplified policy with two parameters, and leads to significant cost reduction as compared to the classical control policy composed of an HPP and a BRP strategy. In addition, this policy is as easy to implement and to manage in practice as the classical HPP and BRP strategy, because it only requires that the inventory level be checked at the scheduled PM times.

\section{References}

Akella, R. \& Kumar, P. R. (1986) Optimal control of production rate in a failure prone manufacturing system. IEEE Transactions on Automatic Control, 31, 116-126.

Archibald, T. W. \& Dekker, R. (1996) Modified block-replacement for multiple-component systems. IEEE Transactions on Reliability, 45, 75-83.

Barlow, R. E. \& Hunter, L. (1960) Optimal preventive maintenance policies. Operations Research, 8, 90-100.

Barlow, R. E. \& Proschan, F. (1965) Mathematical theory of reliability, New York, John Wiley \& Sons.

Berg, M. \& Epstein, B. (1976) A modified block replacement policy. Naval Research Logistics Quarterly, 23, 15-24.

Boukas, E. K. \& Haurie, A. (1990) Manufacturing flow control and preventive maintenance: A stochastic control approach. IEEE Transactions on Automatic Control, 35, 1024-1031.

Chelbi, A. \& Ait-Kadi, D. (2000) Generalized inspection strategy for randomly failing systems subject to random shocks. International Journal of Production Economics, 64, 379-384.

Chelbi, A. \& Ait-Kadi, D. (2004) Analysis of a production/inventory system with randomly failing production unit submitted to regular preventive maintenance. European Journal of Operational Research, 156, 712-718.

Dimitrakos, T. D. \& Kyriakidis, E. G. (2008) A semi-markov decision algorithm for the maintenance of a production system with buffer capacity and continuous repair times. International Journal of Production Economics, 11, 752-762.

Gharbi, A. \& Kenné, J. P. (2000) Production and preventive maintenance rates control for a manufacturing system: an experimental design approach. International Journal of Production Economics, 65, 275-287. 
Gharbi, A., Kenné, J. P. \& Beit, M. (2007) Optimal safety stocks and preventive maintenance periods in unreliable manufacturing systems. International Journal of Production Economics, $107,422-434$.

Hu, J. Q. \& Xiang, D. (1995) Monotonicity of optimal flow control for failure-prone production systems. Journal of Optimization Theory and Applications, 86, 57-71.

Karen, K. Y., Liu, H. \& Yin, G. G. (2003) Stochastic Models and Numerical Solutions for Production Planning with Applications to the paper Industry. Computers and Chemical Engineering, 27, 1693-1706.

Kenné, J. P., Boukas, E. K. \& Gharbi, A. (2003) Control of production and corrective maintenance rates in a multiple-machine, multiple-product manufacturing system. Mathematical and Computer Modelling, 38, 351-365.

Kenné, J. P. \& Gharbi, A. (1999) Experimental design in production and maintenance control problem of a single machine, single product manufacturing system. International Journal of Production Research, 37, 621-637.

Kenné, J. P. \& Nkeungoue, L. J. (2008) Simultaneous control of production, preventive and corrective maintenance rates of a failure-prone manufacturing system. Applied Numerical Mathematics, 58, 180-194.

Ki Ling, C. \& Hausman, W. H. (1997) Joint determination of preventive maintenance and safety stocks in an unreliable production environment. Naval Research Logistics, 44, 257-272.

Kimemia, J. G. \& Gerschwin, S. B. (1983) An algorithm for the computer control of production in flexible manufacturing systems. IIE Transactions, 15, 353-362.

Kushner, H. J. \& Dupuis, P. G. (1992) Numerical methods for stochastic control problems in continuous time, New York, Springer-Verlag.

Kyriakidis, E. G. \& Dimitrakos, T. D. (2006) Optimal preventive maintenance of a production system with an intermediate buffer. European Journal of Operational Research, 168, 75-84.

Montgomery, D. C. (2005) Design and Analysis of Experiments, NY, John Wiley \& Sons.

Pellerin, R., Boulet, J. F., Gharbi, A. \& Kenné, J. P. (2007) Développement d'une politique intégrée de contrôle des taux de production et de maintenance corrective avec diagnostic. Information Systems and Operational Research, 45 (4), 197-208.

Pristker, A. A. B. \& O'reilly, J. J. O. (1999) Simulation with Visual SLAM and Awesim, NY, John Wiley \& Sons.

Rezg, N., Xie, X. \& Mati, Y. (2004) Joint optimisation of preventive maintenance and inventory control in a production line using simulation. International Journal of Production Research, 42, 2029-2046. 
Rezg, N., Dellagi, S. \& Chelbi, A. (2008) Joint optimal control and preventive maintenance policy. International Journal of production Research, 46 (19), 5349-5365.

Rishel, R. (1975) Control of systems with jump Markov disturbances. IEEE Transactions on Automatic Control, 20, 241-244.

Salameh, M. K. \& Ghattas, R. E. (2001) Optimal just-in-time buffer inventory for regular preventive maintenance. International Journal of Production Economics, 74, 157-161.

Van der Duyn Schouten, F. A. \& Vanneste, S. G. (1995) Maintenance optimization of a production system with buffer capacity. European Journal of Operational Research, 82, 323-338. 